\title{
Multicriteria Decision Model to Support the Assignment of Storage Location of Products in a Warehouse
}

\author{
Denilson Dimas da Silva, Natália Veloso Caldas de Vasconcelos, \\ and Cristiano Alexandre Virginio Cavalcante
}

Department of Production Engineering, School of Engineering, Centre for Technology and Geosciences, Federal University of Pernambuco, 50670-901 Recife, PE, Brazil

Correspondence should be addressed to Natália Veloso Caldas de Vasconcelos; nataliaveloso@hotmail.com

Received 23 November 2014; Revised 17 March 2015; Accepted 17 March 2015

Academic Editor: Bo Yu

Copyright ( $) 2015$ Denilson Dimas da Silva et al. This is an open access article distributed under the Creative Commons Attribution License, which permits unrestricted use, distribution, and reproduction in any medium, provided the original work is properly cited.

The storage of products has a strategic role in the supply chain: an efficient organization of products in appropriate locations enables these products to be moved faster inside the warehouse and logistics channel, which increases the speed of delivery and the competitiveness of enterprises. The aim of this paper is to propose a multicriteria decision support model for ranking products and assigning them to warehouse storage locations. During the structuring of the model, the nature of the problem was defined as multicriteria; therefore, the SMARTER and lexicographic methods were applied. The results showed an ordering of products that allowed their strategic location in a hypothetical nonautomated warehouse.

\section{Introduction}

Proper integration of supply chain is one of the major issues in market's competitiveness [1].

This context highlights the importance of warehouse activity, which has received considerable interest in the literature on supply chain management. Warehouse activity is an element of fundamental importance in the integration of the logistics channel, and it is regarded as an extension of production.

The warehouse system function is composed of a set of related activities, namely, receiving, storage/warehousing, order picking (separation of requests), and expedition [2].

This paper addresses the issue of warehouse storage, which one of the main of warehouses and involves guarding products in certain areas of deposit. It aims to achieve an efficient use of space that facilitates the separation of applications and minimizes the cost of order picking [3].

Different criteria, such as size, weight, volume, demand, popularity, cost, and distance, among others, may be used to sort and allocate products to their warehouse positions. When considering all of these criteria simultaneously, selecting the best place to locate products becomes a difficult activity; thus, the ranking and assignment of products to warehouse locations can be understood as a problem of multicriteria decision-making.

In this context, this paper proposes a multicriteria decision model to support the process of assigning storage locations to products in a warehouse. The approach consists of the combined use of two multicriteria methods in a decision-making framework. The procedure of storage location assignment is divided into some steps, as can be seen in Figure 1. First, the best places in the warehouse are identified; then, nondominated products are ranked based on the multicriteria method SMARTER. The assignment of these products is based on their ranking and on the storage capacity of the different places. Finally, dominated products are assigned based on the lexicographic method. An improvement of the effectiveness in warehouse activities related to storage location assignment is expected.

This paper is organized into four sections besides the introduction. The second section described the Storage Location Assignment Problem (SLAP) while, in the third section, a framework to incorporate multicriteria methods is developed. The multicriteria decision support model, which focuses on defining the priority to be given to allocate a product in the warehouse, is divided into three phases: identification and structuring; application; selection and allocation. 


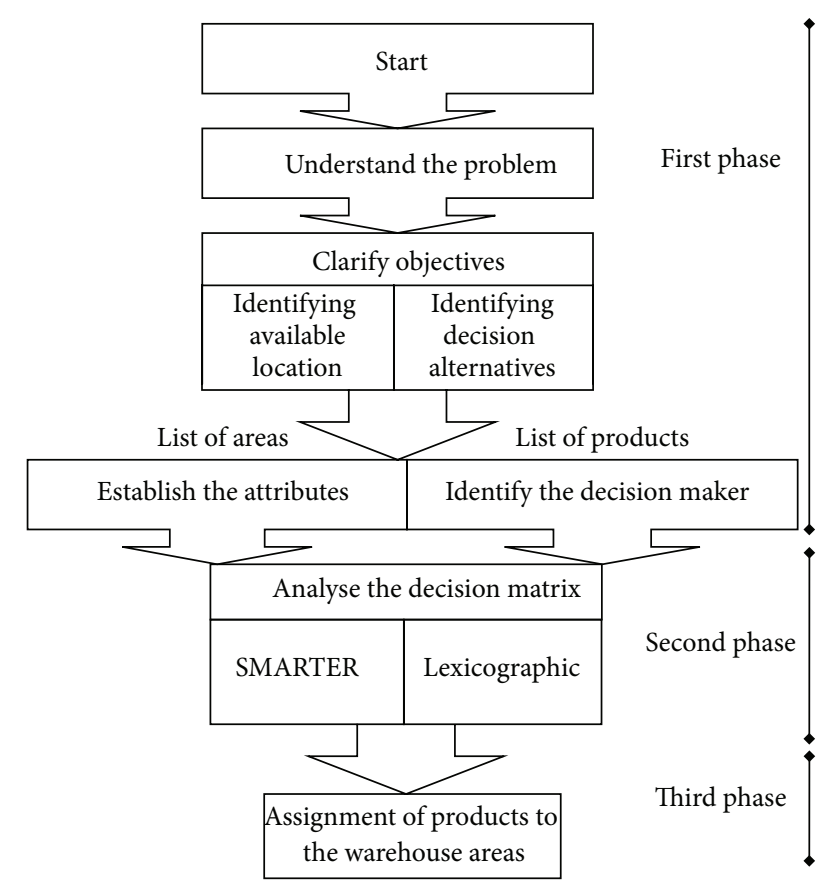

FIGURE 1: Phases of the proposed multicriteria model.

Then, the application section presents an illustrative example and discusses results. Finally, conclusions are drawn on the main aspects related to this paper.

\section{Introduction to Storage Location Assignment Problem}

In the past, warehouses were seen as a system of static installations found along the logistics flow that added operating costs to organizations. In recent decades, the increasing flow of goods and the rapid evolution of technology in materials handling have affected warehouse operations, demanding increased productivity for all activities of the warehouse system.

From an organizational point of view, performances of a storage area are fundamentally based on two variables: the space reserved for material allocation and the time required for their handling [4].

Warehouses began to deliver lower volumes more often with shorter response times and with a much greater variety of products to customers. Thus, the ordering of products and the determination of storage locations became primary challenges of warehouse managers. Petersen et al. [5] mention that, currently, stores perform more minor transactions, stock more products, offer more customization of services and products, and provide more value-added services with less time to process orders and lower margins of error. Many companies try to solve these challenges with better technology, but the solution may lie in a careful analysis of customer orders and products.

2.1. Activities of the Storage Process. Storage is broadly conceptualized, encompassing the entire set of activities ranging from receiving, storage, and order picking to expedition $[2,6-$ 8].

Receiving involves activities such as unloading cargo, checking the quantity and quality of products, and the registration of products [2].

Storage/stockpiling is understood as part of the storage and custody of products for a certain period of time. It is the main function of the warehouse, and it is concerned with the arrangement of goods to achieve high space utilization and facilitate the efficient handling of materials [3].

Petersen et al. [5] discuss order picking as an activity by which a quantity of goods is retrieved from a storage system to satisfy a range of customer orders.

Expedition involves activities, such as loading vehicles, checking requests, preparing shipping documents, and weighing the load to determine the shipping cost [2].

2.2. Allocation of Goods in Warehouses. The Storage Location Assignment Problem (SLAP) establishes how and where goods should be placed inside the warehouse [7].

The question of where to stock the SKUs can be resolved by determining the following: (I) how to sort or classify the SKUs and (II) how to assign ranked SKUs to warehouse locations. According to Petersen et al. [5] also relates that allocation measures are used to determine the order or classification of SKUs and warehouse allocation strategies are used to determine how to assign each SKU to warehouse locations.

An efficient local warehouse assignment policy achieves the following: it reduces the average time of the journey to the warehouse and the time needed for the separation of applications, it evenly distributes the activities of warehouse subsystems, it reduces congestion in aisles, and it enables activities to be better organized [7].

Studies that directly address the attribution of products to warehouse sites, that is, the allocation of local products that allows a greater organization and, in turn, greater warehouse efficiency and that uses the multicriteria approach, are still scarce in the literature.

With this in mind, this study seeks to develop a model that considers different and conflicting criteria for the allocation of products to warehouse storage locations.

Multiple elements that affect the allocation of warehouse space include the characteristics of products, the size and layout of the warehouse system, demand behavior, turnover rates, space requirements, the order of the picking method, and system materials handling, among others [9].

Petersen et al. [5] show the evaluation of warehouse allocation strategies in terms of distance and travel time. The results show better economic performance when the following allocation measures are used: popularity, turnover, and others.

To locate products in their respective places, one uses the set of products to be assigned in a given region, the available locations, and the number of places already occupied. Accordingly, before the location can be selected, the products are evaluated in order to assign an index rating that indicates placement priority. Using the index rating, rules are 
established for the location that can be grouped or dedicated to each particular product.

A poorly conducted review that assigns inconsistent indexes to the products will be reflected in incongruous storage location assignment; instead of contributing to the goals and aspirations of managers, these storage location assignments may result in increased costs and inefficient warehouse activities.

This work will give attention to the process of storage location assignments to products. The structuring of the problem will make it possible to bring a sense of purpose and meaning to some aspects in the process of assigning priority for storage location of goods in a warehouse, because the purpose is to establish a decision-making model that allows offering a systematic treatment of the structural development of the problem in question.

The definition of the storage location assignments of products is a characteristic of multicriteria decision problems because managerial preferences between the criteria will lead to a final decision of the best use of the available space for the products to be positioned in different locations in a warehouse. Much effort has been committed to the development of theories and methodologies that contribute to the assessment of preferences and, in turn, to evaluating the instance of a decision.

To Cavallaro and Ciraolo [10] the use of decision-making tools under a multicriteria approach is intended to aid the decision maker in creating a set of relations between various alternatives. A decision support system can be defined as an interactive system that is able to produce data and information that promote understanding for a given application domain in order to provide useful assistance in resolving complex and ill-defined problems.

This study will initially concentrate on the synthesis criterion approach using the SMARTER method for the purpose of building a formalized decision-making model for the development of a decision support process that may be coherent with the objectives and the value system of the actors; this will be followed by building a structure for the use of the lexicographic method.

SMARTER is a variant of the SMART method that is characterized by being applicable to problems of a compensatory nature in that it makes use of the additive aggregation procedure for alternative criteria.

Edwards and Barron [11] stated that the SMARTER method is conducted through the following phases.

Phase 1: purposes and decioson makers-the decision maker is identified, and the purposes of eliciting the value problem under consideration are placed; thus, it comprises a specification of the nature and structure of data to be elicited.

Phase 2: getting the structure of attributes-the objectives are determined together with the attributes.

Phase 3: establishment of alternatives.

Phase 4: construction of the matrix of consequencesto obtain the matrix with all of the consequences for each alternative according to each criterion.

Phase 5: elimination of dominated alternatives.

Phase 6: building the assessment matrix-which constitutes intracriteria evaluation, thus obtaining the value functions of all criteria. The task is to evaluate the best and the worst results, you will have extreme values on the scale of 0 and 1 , and the consequences with these two extremes receive proportional amounts from the linear relationship.

Phase 7: the procedure uses the swing to sort the criteria. It is included in the evaluation matrix's hypothetical alternative that has the worst performance of all criteria, taking the value of 0 . The following questions are posed. (1) When choosing the alternative with the worst outcome, if you could improve the performance of this alternative in only one of the criteria for the maximum value, what criterion would you choose? The criterion that chosen is the one that will have the highest value of a constant scale. (2) If you can also improve the performance of the alternative in one of the remaining criteria, which criterion would you choose? The criterion chosen will have the second highest constant scale. The questioning continues until all criteria are evaluated.

Phase 8: obtaining the weights. Barron and Barrett [12] developed the Ranking Order Centroid (ROC), justifying it as a procedure to transform the order of criteria into weights. That is, the criteria that are arranged in the first positions will receive a larger weight than the subsequent ones.

Phase 9: execution of the additive aggregation and choice. Values are calculated for each alternative with the additive aggregation procedure in accordance with the scale constants (weights) obtained in the previous stage. The final step is the sensitivity analysis.

Edwards and Barron [11] have shown that, after you know the $u(x)$ for each value dimension, the next step is to determine how to aggregate the vector values of $u(x)$ on a scale. The authors state that additive aggregation is a simple way to do this by means of an additive model. As such, we get the following model: $U_{h}=\sum_{k=1}^{k} w_{k} u_{h}\left(x_{h k}\right)$, where $h$ is the index of alternatives, $k$ is an index of the value dimensions, $u_{h}\left(x_{h k}\right)$ are the one-dimensional value function, and $w_{k}$ are the weights for each value dimension. The sum of the weights must be equal to 1 .

2.3. The Lexicographic Method. According to Arunkumar et al. [13] in the lexicographic method, the designer ranks the objectives in order of importance. The optimum solution $X^{*}$ is then found by minimizing the objective functions, starting with the most important and proceeding according to the order of importance of the objectives.

In the lexicographic method only the order of performance of the alternatives and the importance of the criteria are taken into account [14].

For Munda [15], a preference relation is noncompensatory if no trade-off occurs, and it is compensatory otherwise.

In the next section, the structural elements of the proposed multicriteria model will be presented, specifying all of the components of each phase, which will serve as support for the numerical application of the problem of ranking and allocating products in storage locations.

\section{Structuring the Product Allocation Model}

The multicriteria decision support model, which focuses on the definition of a product's positioning priority in the 
warehouse, is divided into three phases: identification and structuring, application, and selection and allocation.

Phase 1 enables defining the problem, the objectives, the alternatives, the criteria, and the decision maker, corresponding to the first three steps of the SMARTER method. Phase 2 allows the numerical application with the dominated and nondominated alternatives using steps four to nine of the SMARTER method and the lexicographic method, respectively. Phase 3 defines the ranking of alternatives to prioritize the positioning of products in the warehouse.

The determination of the set of alternatives outlined in the model can be represented as follows: $A=\left(P_{1}, P_{2}, P_{3}, \ldots, P_{n}\right)$. This set corresponds to the organization of all distinct products according to their characteristics in the warehouse, which, when added together, result in the complete set of products that a business organization needs to store.

As far as the design of the warehouse goes, a simplified model of the hypothetical warehouse was established with a layout divided into several categories for the allocations of the products. The proposed warehouse design emphasizes the horizontal dimension.

The criteria were established according to the relevant literature, namely,

(1) population: a quantitative attribute that expresses the average number of consumers served by each product in a monthly period; the delivery deadline of the products to consumers also depends on how they are organized inside the warehouse;

(2) turnover: a quantitative attribute that corresponds to the quantity of products shipped monthly, in units; it represents the demand for products extracted from their locations up to the I/O point; that is, it is the amount of SKUs shipped; this criterion is related to order picking and accessibility costs, with the products being located as close as possible to the I/O point;

(3) volume (length, width, and height)/space: a quantitative attribute referring to the size of the product, given in cubic meters $\left(\mathrm{m}^{3}\right)$; it should be noted that the volume of the product itself reflects the space that will be needed for its allocation; this criterion is therefore directly linked to the cost of space.

The decision maker is the warehouse manager. The decision maker is important in this process because it is through him that the elicitation process of model parameters is achieved for the construction of the problem's structure. However, this study took into account the ROC procedure for weighting by which one can transform the order information of criteria (part 1 of step 7 of the SMARTER method) into weights without needing any additional evaluation from the decision maker.

\section{Application and Results}

4.1. Phase 1: Structuring the Problem. By structuring the model according to the sequence of the first three steps of the SMARTER method, the problem could be defined: arranging a mix of products and allocating them in locations inside the warehouse from the perspective of multicriteria. The objectives and most relevant criteria were also established through the literature, in addition to the decision maker, who in this study is the warehouse manager.

4.2. Phase 2: Numerical Application. The following application is not an actual case, but it employs realistic data based on a context and the structure of relations between the considered variables. This application will employ the SMARTER and lexicographic methods. The sequence of steps allows the model to be adapted according to the problem's peculiarities, for example, by including or excluding criteria and alternatives.

4.2.1. Using the Multicriteria SMARTER Method for Nondominated Alternatives. For each value function, physical values were included corresponding to each alternative per evaluation criterion. The respective scores were formalized in conjunction with the decision maker in order to develop the matrix of alternatives per criterion. This process corresponds to step 4 of the SMARTER method. It is worth emphasizing that the highlighted alternatives are those that were nondominated, which means that the dominated alternatives were eliminated for use in SMARTER, according to step 5 of the SMARTER method. The matrices are shown in Table 1.

We arrive at step 6 assuming that the value function for each criterion is linear. The result can be seen in Table 2, where the values of the one-dimensional functions of alternatives per criterion were normalized using the procedure where

$$
v_{j}^{\prime}\left(a_{i}\right)=\frac{\left[v_{j}\left(a_{i}\right)-\operatorname{Min} v_{j}\right]}{\left[\operatorname{Max} v_{j}-\operatorname{Min} v_{j}\right]} .
$$

After establishing the matrices, the elicitation process for determining the relative importance of criteria was continued with the decision maker. The following questions were posed to the decision maker: "Supposing the choice of one alternative, which criterion should be chosen to improve the performance of this alternative in only one of the criteria to a maximum value?" and "Which criterion should be adopted to improve the performance of the same alternative in only one of the criteria, except for the previously chosen criterion?"

This process is called part 1 of the swing step 7 of the SMARTER method, and it is used for all of the criteria. The first criterion chosen will have the highest weight (scale constants), the next criterion will have the second highest weight, and so on. All criteria were evaluated and were based on the information from the decision maker, and the sequence of the relative importance of the criteria was established.

To establish the weights of the criteria, that is, to transform the sequence information of the criteria into weights, the ROC procedure was used, which is step 8 of SMARTER. ROC enables the weights of the criteria to be set without an additional evaluation by the decision maker. It establishes the weights of the criteria according to their relative importance.

After all of the procedures were carried out to aggregate the one-dimensional functions of alternatives per criterion, 
TABLE 1: Matrix of alternatives by criteria.

\begin{tabular}{|c|c|c|c|}
\hline \multirow{2}{*}{ Alternatives } & \multicolumn{3}{|c|}{ Criteria } \\
\hline & Population (q) & Turnover (q) & Volume (unid $/ \mathrm{m}^{3}$ ) \\
\hline$P_{1}$ & 150 & 300 & 1 \\
\hline$P_{2}$ & 301 & 3500 & 1,6 \\
\hline$P_{3}$ & 150 & 70 & 1,8 \\
\hline$P_{4}$ & 158 & 400 & 2,2 \\
\hline$P_{5}$ & 50 & 150 & 1,8 \\
\hline$P_{6}$ & 500 & 200 & 1 \\
\hline$P_{7}$ & 330 & 495 & 0,8 \\
\hline$P_{8}$ & 120 & 160 & 1,6 \\
\hline$P_{9}$ & 90 & 80 & 0,3 \\
\hline$P_{10}$ & 237 & 400 & 1,9 \\
\hline$P_{11}$ & 342 & 500 & 1,2 \\
\hline$P_{12}$ & 62 & 700 & 0,8 \\
\hline$P_{13}$ & 62 & 645 & 2 \\
\hline$P_{14}$ & 312 & 111 & 1,8 \\
\hline$P_{15}$ & 435 & 2000 & 0,8 \\
\hline$P_{16}$ & 123 & 2212 & 2,3 \\
\hline$P_{17}$ & 234 & 4031 & 0,5 \\
\hline$P_{18}$ & 208 & 120 & 0,7 \\
\hline$P_{19}$ & 40 & 634 & 1,8 \\
\hline$P_{20}$ & 118 & 654 & 0,6 \\
\hline$P_{21}$ & 340 & 4677 & 1,7 \\
\hline$P_{22}$ & 126 & 2300 & 0,5 \\
\hline$P_{23}$ & 110 & 1234 & 0,8 \\
\hline$P_{24}$ & 190 & 2456 & 1,5 \\
\hline$P_{25}$ & 400 & 51 & 2,5 \\
\hline$P_{26}$ & 151 & 2700 & 1,2 \\
\hline$P_{27}$ & 32 & 12 & 2,4 \\
\hline$P_{28}$ & 322 & 40 & 2,2 \\
\hline$P_{29}$ & 170 & 76 & 0,7 \\
\hline$P_{30}$ & 175 & 3000 & 1,1 \\
\hline
\end{tabular}

TABLE 2: Normalized matrix with nondominated alternatives by criteria.

\begin{tabular}{lccc}
\hline \multirow{2}{*}{ Alternatives } & \multicolumn{3}{c}{ Criteria } \\
& Population (q) & Turnover (q) & Volume (unid $\left./ \mathrm{m}^{3}\right)$ \\
\hline$P_{2}$ & 0,51 & 0,74 & 0,07 \\
$P_{6}$ & 1,00 & 0,03 & 0,50 \\
$P_{7}$ & 0,59 & 0,09 & 0,64 \\
$P_{9}$ & 0,00 & 0,00 & 1,00 \\
$P_{15}$ & 0,84 & 0,42 & 0,64 \\
$P_{17}$ & 0,35 & 0,86 & 0,86 \\
$P_{21}$ & 0,61 & 1,00 & 0,00 \\
$P_{22}$ & 0,09 & 0,48 & 0,86 \\
\hline
\end{tabular}

the calculation to obtain the overall value of each alternative could finally be performed, thus enabling the choice of alternatives. In this way, we arrive at step 9 of the SMARTER method.
Table 3 summarizes the matrix that will be used for the scope of the results of the additive aggregation using the multicriteria SMARTER method. The criteria are positioned according to the order of importance (from left to right) and the respective weights of the criteria in addition to the calculation of the overall multiple attribute value of each alternative per criterion.

Based on the values of the overall multiple attribute functions of each alternative, it is possible to arrange these alternatives from best to worst.

The values of each alternative were calculated through the additive aggregation procedure with scale constants, resulting in overall values. An arrangement of alternatives according to their respective scores in the priority setting and storage location allocation process is presented in Table 4 .

A sensitivity analysis for this ranking was made in order to observe the influence of the scale constants on the final raking of the alternatives.

The values of the scale constants of the criteria were modified to check the sensitivity of the model with respect to the criteria. The weights of the criteria were modified by 10 and $15 \%$ on the lower and upper limits, and the differences were distributed proportionally to the other criteria. Some observations were made.

(i) By varying the scale constant of criterion population, changes occurred in the first five positions of the ranking, between alternatives $P_{15}, P_{6}, P_{21}, P_{2}$, and $P_{17}$. It is worth noting that the first position was between $P_{15}$ and $P_{6}$ while the other positions remained unchanged throughout the analysis.

(ii) By varying the scale constant of criterion turnover, changes occurred only in the first three positions of the ranking between alternatives $P_{15}, P_{6}, P_{21}$.

(iii) No alteration was observed by varying the scale constant of criterion volume: the ranking remained the same for the range of variation.

For changes involving the ROC, by changing the ordering of the criteria, the following observations could be made:

all alternatives underwent changes in all analyses; the alternatives oscillated between several positions.

4.2.2. Using the Lexicographic Method for Dominated Alternatives. The lexicographic method enables the prioritization of alternatives that were discarded by the multicriteria SMARTER method because they proved to be dominated.

Based on the same criteria and scores of the alternatives addressed in the structure of the SMARTER method, the lexicographic method begins with the decision maker ranking the criteria by order of importance. The alternatives are sorted by their value for the most important criterion. For the subset of alternatives in which there were ties, the alternatives are ordered by the value for the second most important criterion, and so on. See Table 5.

4.3. Phase 3: Determining the Priority of the Product for Positioning in the Warehouse. The discussed application 
TABLE 3: Alternative matrix by criteria, weights, and calculation of the multiple attribute value function.

\begin{tabular}{lcccc}
\hline & & \multicolumn{2}{c}{ Criteria } \\
Alternatives & Population (q) & Turnover (q) & 0,11 & Multiple attribute value \\
\hline$P_{2}$ & 0,61 & 0,28 & 0,01 & 0,53 \\
$P_{6}$ & 0,31 & 0,21 & 0,06 & 0,67 \\
$P_{7}$ & 0,61 & 0,01 & 0,07 & 0,45 \\
$P_{9}$ & 0,36 & 0,03 & 0,11 & 0,11 \\
$P_{15}$ & 0,00 & 0,00 & 0,07 & 0,70 \\
$P_{17}$ & 0,51 & 0,12 & 0,09 & 0,55 \\
$P_{21}$ & 0,21 & 0,24 & 0,00 & 0,65 \\
$P_{22}$ & 0,37 & 0,28 & 0,09 & 0,28 \\
\hline
\end{tabular}

TABLE 4: Ranking of nondominated alternatives.

\begin{tabular}{lcc}
\hline Ranking of alternatives & Multiple attribute value \\
\hline 1 & $P_{15}$ & 0,70 \\
2 & $P_{6}$ & 0,67 \\
3 & $P_{21}$ & 0,65 \\
4 & $P_{17}$ & 0,55 \\
5 & $P_{2}$ & 0,53 \\
6 & $P_{7}$ & 0,45 \\
7 & $P_{22}$ & 0,28 \\
8 & $P_{9}$ & 0,11 \\
\hline
\end{tabular}

results in the prioritization of products so that they can be stored inside the warehouse. Thus, the company will possess information that enables a more efficient organization of the warehouse, which can enhance competitiveness in the market. In other words, products (alternatives) are stored in appropriate locations, facilitating fast deliveries and the satisfaction of consumer demands.

This study addresses the problem of establishing storage location assignments for products within a warehouse. The storage location assignment of the products enables the establishment of distribution guidelines for the products inside the warehouse, contributing to a more efficient storage location process.

This ranking works as an indicator for allocating products. The most efficient allocation is achieved by ranking the alternatives. The storage standard adopted is the one obtained based on the descending order of the overall values from the SMARTER method and the prioritization established by the lexicographic method.

With regard to the products that were applied in the structure of the SMARTER method, those that obtained high overall values contributed significantly to the competitive positioning of the organization. They should therefore be stored in locations closer to the I/O point, contributing to cost reductions in order picking and minimizing delays in product deliveries. The products with lower overall scores will be less requested and therefore should be stored in more distant locations from the I/O point.

With regard to the products embedded in the structure of the lexicographic method, they were located in places closer

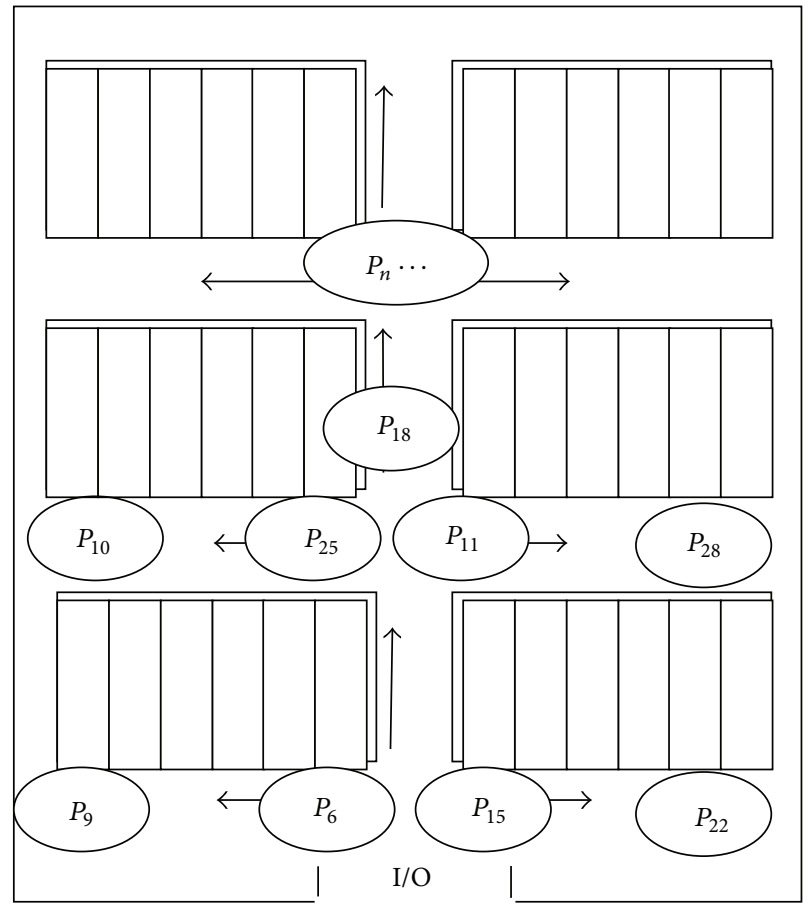

FIgURE 2: Allocations.

to the back of the warehouse according to the order of priority given by the application of the method.

The allocation of products to storage locations follows the distribution shown in Figure 2.

The execution was undertaken in a hypothetical warehouse with well-defined characteristics. If necessary and desired, the model may be extended for executions in warehouses with similar characteristics.

As previously mentioned, the sequence of steps of the methods used allows the model to be adapted according to the particularities of the problem, such as, for instance, the inclusion or the exclusion of criteria and alternatives.

The problematic of arranging the products may be applied in other cases and not only for storage location assignment of the products. 
TABLE 5: Ranking of dominated alternatives.

\begin{tabular}{|c|c|c|c|c|c|}
\hline Ranking & Alternatives & $\begin{array}{c}\text { Population (q) } \\
0,61 \\
\end{array}$ & $\begin{array}{c}\text { Turnover (q) } \\
0,28 \\
\end{array}$ & $\begin{array}{c}\text { Volume (unid } / \mathrm{m}^{3} \text { ) } \\
0,11 \\
\end{array}$ & Multiple attribute value \\
\hline 9 & $P_{11}$ & 0,84 & 0,16 & 0,68 & 0,63 \\
\hline 10 & $P_{25}$ & 1,00 & 0,01 & 0,00 & 0,61 \\
\hline 11 & $P_{30}$ & 0,39 & 1,00 & 0,74 & 0,60 \\
\hline 12 & $P_{24}$ & 0,43 & 0,82 & 0,53 & 0,55 \\
\hline 13 & $P_{26}$ & 0,32 & 0,90 & 0,68 & 0,52 \\
\hline 14 & $P_{14}$ & 0,76 & 0,03 & 0,37 & 0,51 \\
\hline 15 & $P_{28}$ & 0,79 & 0,01 & 0,16 & 0,50 \\
\hline 16 & $P_{10}$ & 0,56 & 0,13 & 0,32 & 0,41 \\
\hline 17 & $P_{18}$ & 0,48 & 0,04 & 0,95 & 0,41 \\
\hline 18 & $P_{16}$ & 0,25 & 0,74 & 0,11 & 0,37 \\
\hline 19 & $P_{23}$ & 0,21 & 0,41 & 0,89 & 0,34 \\
\hline 20 & $P_{29}$ & 0,38 & 0,02 & 0,95 & 0,34 \\
\hline 21 & $P_{20}$ & 0,23 & 0,21 & 1,00 & 0,31 \\
\hline 22 & $P_{1}$ & 0,32 & 0,10 & 0,79 & 0,31 \\
\hline 23 & $P_{4}$ & 0,34 & 0,13 & 0,16 & 0,26 \\
\hline 24 & $P_{3}$ & 0,32 & 0,02 & 0,37 & 0,24 \\
\hline 25 & $P_{12}$ & 0,08 & 0,23 & 0,89 & 0,21 \\
\hline 26 & $P_{8}$ & 0,24 & 0,05 & 0,47 & 0,21 \\
\hline 27 & $P_{13}$ & 0,08 & 0,21 & 0,26 & 0,14 \\
\hline 28 & $P_{19}$ & 0,02 & 0,21 & 0,37 & 0,11 \\
\hline 29 & $P_{5}$ & 0,05 & 0,05 & 0,37 & 0,08 \\
\hline 30 & $P_{27}$ & 0,00 & 0,00 & 0,05 & 0,01 \\
\hline
\end{tabular}

The study was made based on a single scenario. Therefore, the definitions of the problem were determined and illustrated for this scenario. However, the decision framework may be applied in other scenarios, for which only a few adaptations are needed.

The relevance of the results is related to the enrichment provided by the multicriteria methods for meeting the decision maker's demands with regard to building a broader perspective about the options in a SLAP problem. In practical terms, a more appropriate product positioning in a warehouse may result in companies achieving a competitive advantage.

\section{Conclusions}

This model contributes a multicriteria methodology that enables the ranking of products that can be allocated within the warehouse in a descending order from the best to worst options for each product.

The ranking of alternatives using the SMARTER and lexicographic methods is therefore considered a storage standard. The products with the highest overall values should be allocated closest to the I/O (input and output) point, and this allocation rationale should be followed until all products are properly stored.

It should be emphasized that the products that are not included in the SMARTER method were those presented as dominated alternatives. They should therefore be the ones with the smallest significant impact on the objectives proposed in the model and allocated in more distant locations from the I/O point according to the lexicographic method. Thus, by combining these methods, in the routine of a warehouse, the decision maker is able to deal not only with the most important products, but also with those that are dominated.

For further research, we suggest the evaluation of the main elements for implementing the software of the decisionmaking framework, the use of other multicriteria methods, and, finally, proposing new criteria in a different scenario.

\section{Conflict of Interests}

The authors declare that there is no conflict of interests regarding the publication of this paper.

\section{References}

[1] J. R. Pérez-Gallardo, B. Hernández-Vera, C. G. Moras Sánchez et al., "Methodology for supply Chain integration: a case study in the Artisan Industry of footwear," Mathematical Problems in Engineering, vol. 2014, Article ID 508314, 15 pages, 2014.

[2] B. Rouwenhorst, B. Reuter, V. Stockrahm, G. J. van Houtum, R. J. Mantel, and W. H. M. Zijm, "Warehouse design and control: framework and literature review," European Journal of Operational Research, vol. 122, no. 3, pp. 515-533, 2000.

[3] J. Gu, M. Goetschalckx, and L. F. McGinnis, "Research on warehouse operation: a comprehensive review," European Journal of Operational Research, vol. 177, no. 1, pp. 1-21, 2007. 
[4] C. Battista, A. Fumi, F. Giordano, and M. M. Schiraldi, "Storage location assignment problem: implementation in a warehouse design optimization tool," in Proceedings of the Conference Breaking Down the Barriers between Research and Industry, September 2011.

[5] C. G. Petersen, C. Siu, and D. R. Heiser, "Improving order picking performance utilizing slotting and golden zone storage," International Journal of Operations \& Production Management, vol. 25, no. 10, pp. 997-1012, 2005.

[6] J. P. Van Den Berg, "A literature survey on planning and control of warehousing systems," IIE Transactions, vol. 31, no. 8, pp. 751$762,1999$.

[7] J. P. van den Berg and W. H. M. Zijm, "Models for warehouse management: classification and examples," International Journal of Production Economics, vol. 59, no. 1-3, pp. 519-528, 1999.

[8] M.-C. Chen and H.-P. Wu, "An association-based clustering approach to order batching considering customer demand patterns," Omega, vol. 33, no. 4, pp. 333-343, 2005.

[9] F. T. S. Chan and H. K. Chan, "Improving the productivity of order picking of a manual-pick and multi-level rack distribution warehouse through the implementation of class-based storage," Expert Systems with Applications, vol. 38, no. 3, pp. 2686-2700, 2011.

[10] F. Cavallaro and L. Ciraolo, "A multicriteria approach to evaluate wind energy plants on an Italian island," Energy Policy, vol. 33, no. 2, pp. 235-244, 2005.

[11] W. Edwards and F. H. Barron, "SMARTS and SMARTER: improved simple methods for multiattribute utility measurement," Organizational Behavior and Human Decision Processes, vol. 60, no. 3, pp. 306-325, 1994.

[12] F. H. Barron and B. E. Barrett, "Decision quality using ranked attribute weights," Management Science, vol. 42, no. 11, pp. 15151523, 1996.

[13] N. Arunkumar, L. Karunamoorthy, S. Anand, and T. R. Babu, "Linear approach for solving a piecewise linear vendor selection problem of quantity discounts using lexicographic method," International Journal of Advanced Manufacturing Technology, vol. 28, no. 11-12, pp. 1254-1260, 2006.

[14] S.-Y. Liu and S.-C. Chi, "Fuzzy multiple attribute decision making approach using modified lexicographic method," in Proceedings of the IEEE International Conference on Systems, Man and Cybernetics, pp. 19-24, October 1995.

[15] G. Munda, "Measuring sustainability: a multi-criterion framework," Environment, Development and Sustainability, vol. 7, no. 1, pp. 117-134, 2005. 


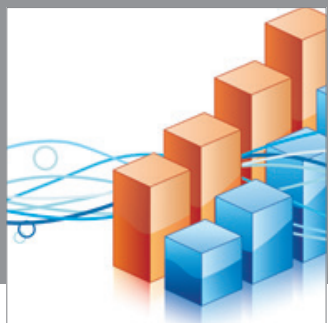

Advances in

Operations Research

mansans

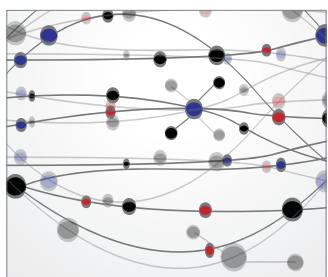

The Scientific World Journal
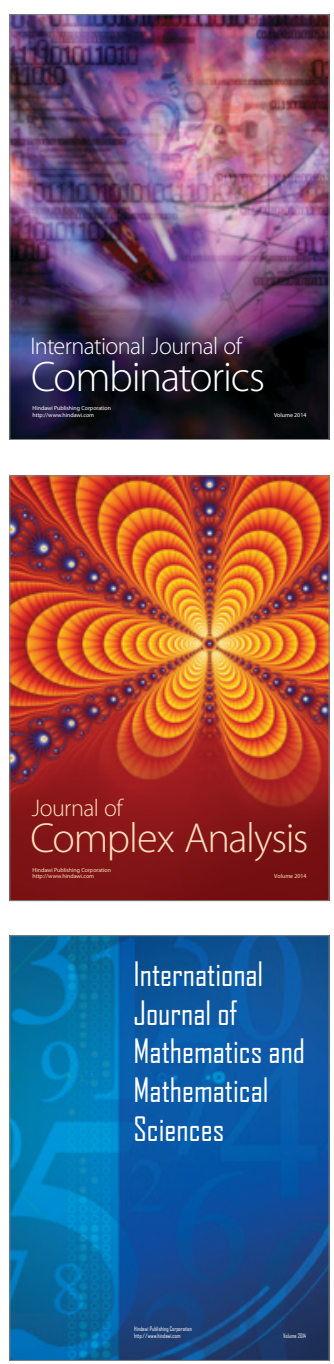
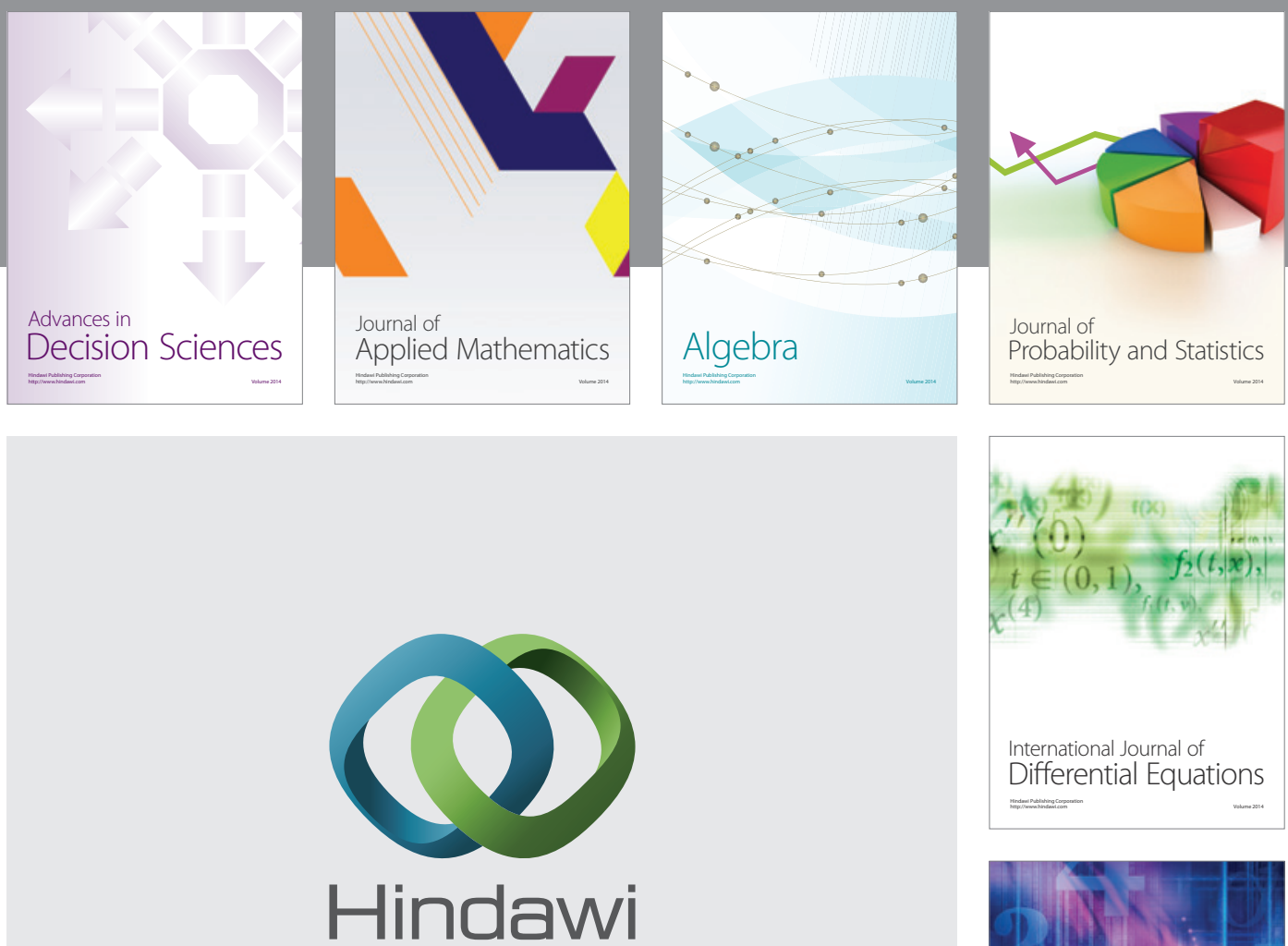

Submit your manuscripts at http://www.hindawi.com
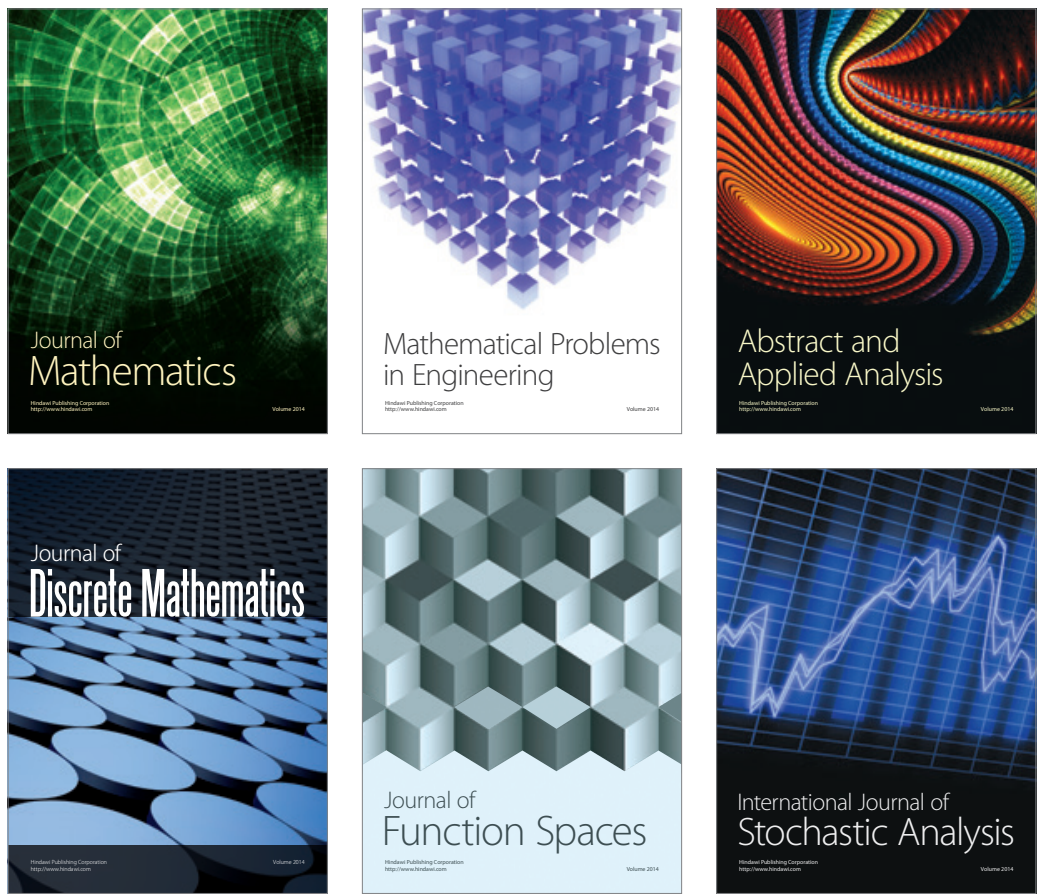

Journal of

Function Spaces

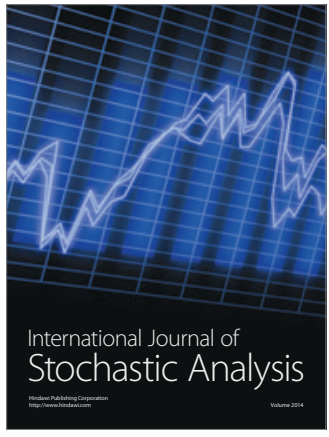

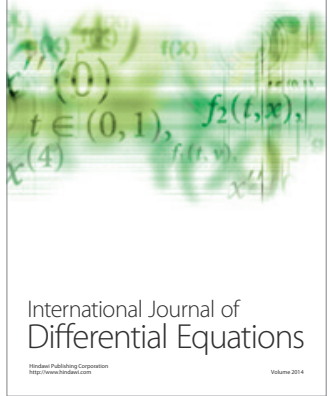
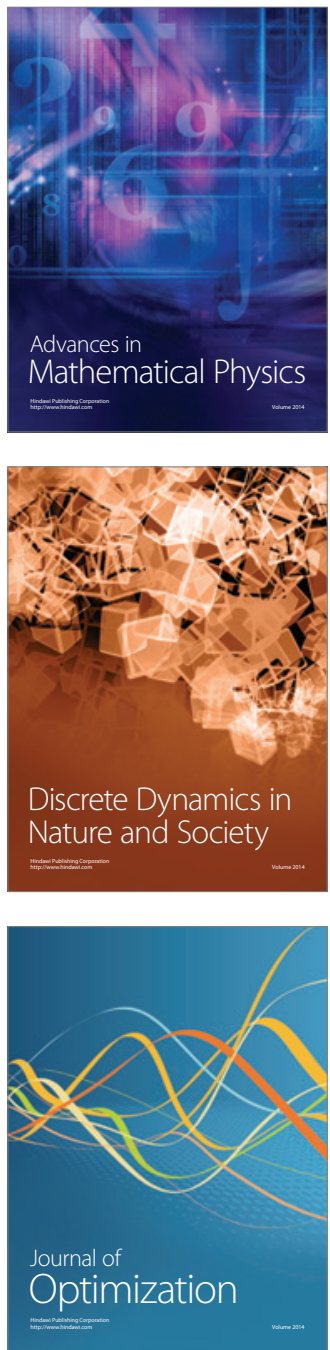